\title{
Regiodivergent condensation of 5-alkoxycarbonyl-1H-pyrrol- 2,3-diones with cyclic ketazinones en route to spirocyclic scaffolds
}

\author{
Alexey Yu. Dubovtsev ${ }^{1}$, Maksim V. Dmitriev ${ }^{1}$, Andrey N. Maslivets ${ }^{* 1}$ \\ and Michael Rubin ${ }^{\star 2,3}$
}

\section{Full Research Paper}

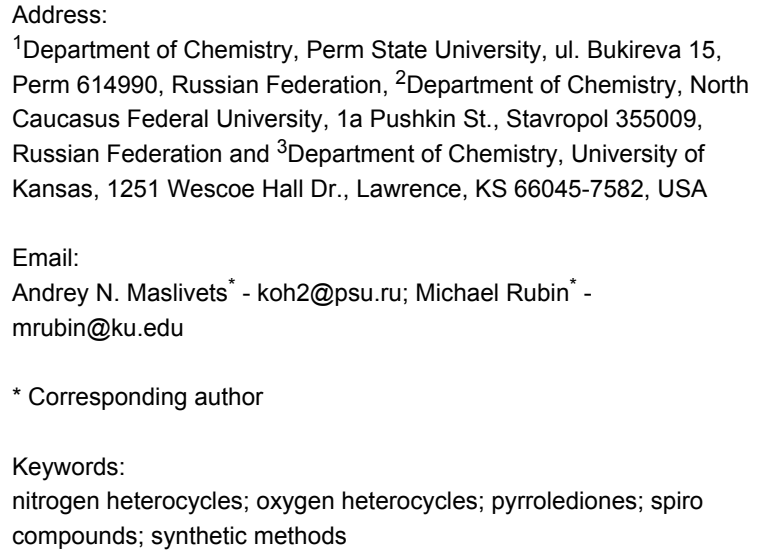

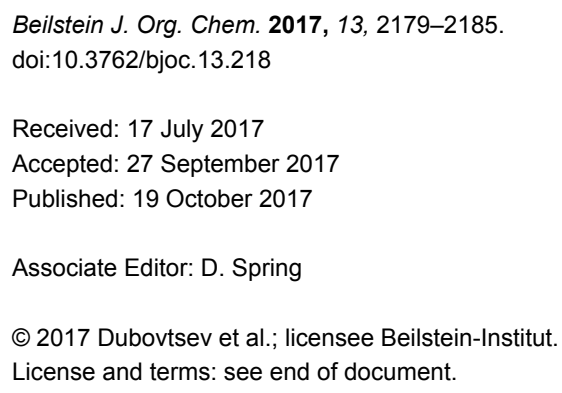

\begin{abstract}
The condensation of 5-alkoxycarbonyl- $1 H$-pyrrolediones with cyclic ketazinones was systematically investigated. It was discovered that the regioselectivity of this reaction can be easily swapped between two alternative directions affording derivatives of partially hydrogenated indole or benzofurane. The control of this regioselectivity is efficiently governed by steric effects at the hydrazone moiety of the ketazinone reagent.
\end{abstract}

\section{Introduction}

Molecular structures based on partially or exhaustively hydrogenated indole and benzofuran cores are omnipresent in nature. Both types of ring systems are found in a variety of important biologically active natural products [1-20], which continue to remain in the focus of attention for many research groups worldwide as targets for total synthesis and to serve as inspiration for exercises in drug design. Although many preparative methods of assembly of these structural units have been demonstrated, the development of new efficient and highly selective synthetic tools is always desired. From this prospective, we have become greatly interested in the chemistry of $1 \mathrm{H}$-pyrrole2,3-diones, polyfunctional building blocks that have great potential for the synthesis of heterocyclic structures. Indeed, these highly electrophilic cyclic vinylogous amides are known 
to undergo facile nucleophilic additions [21-25], sometimes accompanied with subsequent pericyclic rearrangements [2633]. Not surprisingly, these versatile synthons have been successfully employed in the target-oriented synthesis of pyrrolebased natural alkaloids [34-38]. Herein we wish to report a new synthetic route towards spirocyclic scaffolds possessing partially hydrogenated indole or benzofuran cores. The featured approach is based on the highly efficient regiodivergent spirocondensation of 5-alkoxycarbonyl-1 $H$-pyrrole-2,3-diones (serving as 1,2-bis-electrophiles) with cyclic ketazinones (serving as either 1,3-C,N-or 1,3-C,O-bis-nucleophiles).

\section{Results and Discussion}

Previously, we demonstrated a convenient approach towards spiro[indole-3,2'-pyrroles] 3 based on catalyst-free cyclocondensation of six-membered cyclic enamines $\mathbf{1}$ (vinylogous secondary amides) with 5-methoxycarbonyl- $1 H$-pyrrolediones 2 [39-41]. This transformation involved the Michael addition of an enamine to the $\alpha, \beta$-unsaturated carbonyl fragment of a pyrroledione and subsequent 5-exo-trig intramolecular nucleophilic attack of the amine moiety on the ester substituent (Scheme 1). Interestingly, it seems that the substitution at the nitrogen atom in structure $\mathbf{1}$ is very crucial in governing the desired reactivity. Indeed, our previous attempts to expand the substrate scope to include "vinylogous primary amides" 1a resulted in the discovery of an alternative mechanistic pathway. Apparently, in this case the primary amine moiety in intermediate 4 preferred a nucleophilic attack on the keto function, affording bridged hemiaminal structures $\mathbf{5}$ as kinetic products (Scheme 1) [42]. Upon extended heating, however, recyclization into the thermodynamically more favorable "normal" spirocyclic products $\mathbf{3 a}$ occurs.

It should be further noted that in contrast to reactions of enamines, which readily provide the corresponding adducts with pyrrolediones in the absence of catalysts, the similar transformation involving enols 6 (vinylogous carbonates and carbamates) normally requires more forcing conditions, but usually can be facilitated by addition of catalytic amounts of organic base (Scheme 2) [43].

Interestingly, we figured out that the presence of heteroatom $\mathrm{X}$ in the structure of enol $\mathbf{6}$ is important for the normal course of the spirocyclization reaction. Our multiple attempts to carry out this transformation with the participation of enolates generated from cyclohexane-1,3-diones 8 (vinylogous carboxylates) in the presence of bases were unsuccessful. This reaction did not proceed in the presence of weak bases (such as tertiary amines), while the use of stronger bases (hydroxides or alkoxides) caused decomposition of the base-sensitive $1 H$-pyrrole-2,3dione moiety 9 . An attempt to perform the reaction in the pres-
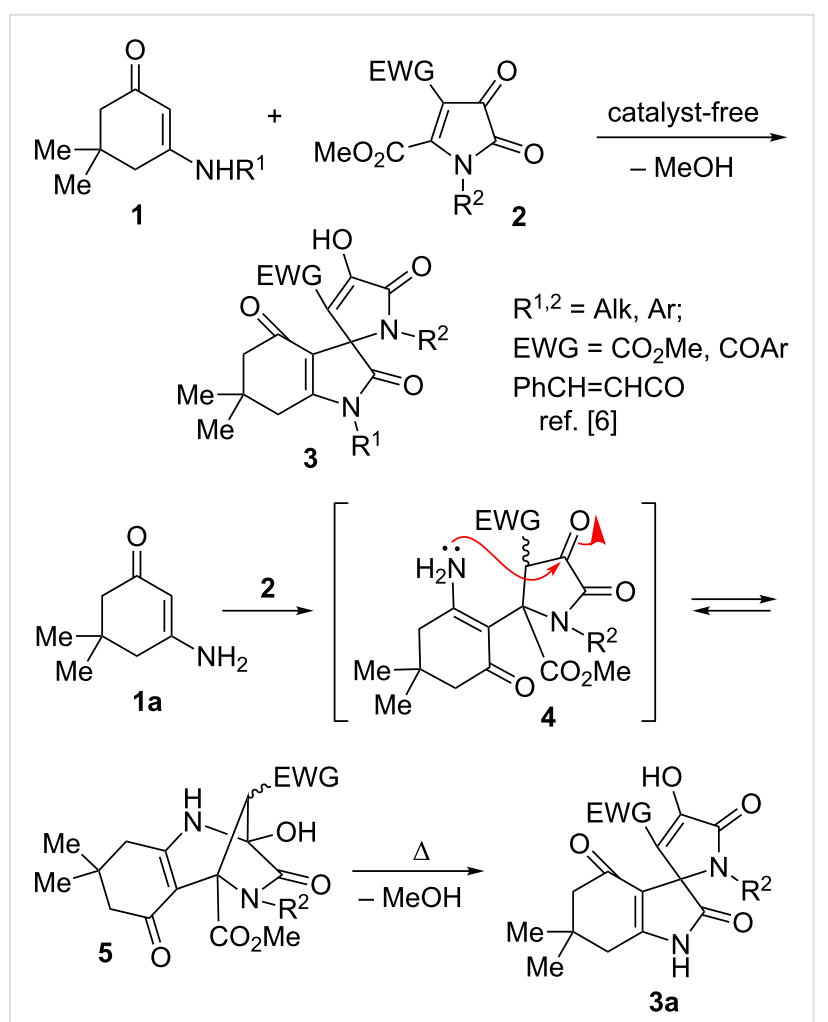

ref. [7]

Scheme 1: Spirocyclization of enamines with 5-methoxycarbonyl-1Hpyrrolediones.

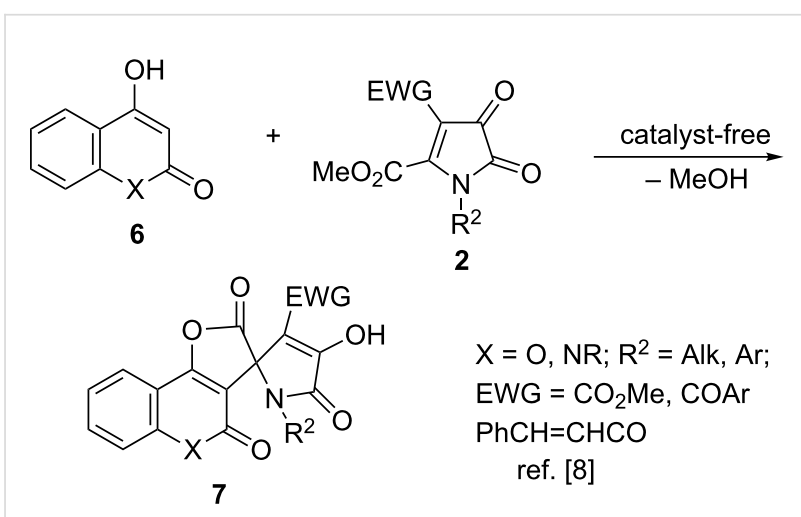

Scheme 2: Non-catalyzed spirocyclization of enoles (vinylogous carbonates and carbamates) with 5-methoxycarbonyl-1H-pyrrolediones.

ence of catalytic amounts of Brønsted acid (TsOH) also did not provide the spirocyclic products. Instead, bridged 1,3-oxazepine products 12 were formed in marginal yields, resulting from an initial aldol reaction involving the carbonyl group at C-3 of pyrroledione 9 and a subsequent intramolecular 6-endo-trig $O$-nucleophilic attack of the enol species at a conjugate unsaturated ketone moiety in the five-membered ring of intermediate 11 (Scheme 3). 


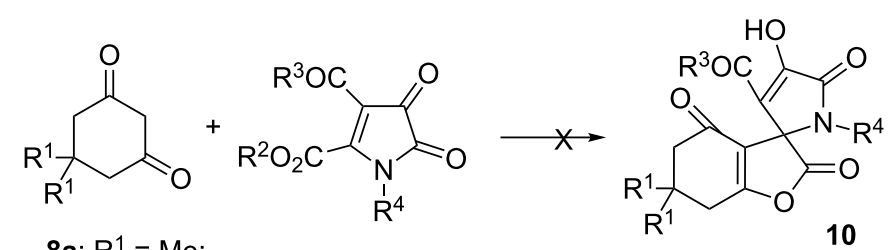

8a: $R^{1}=M e ;$

8b: $\mathrm{R}^{1}=\mathrm{H}$

9a: $R^{2}=M e, R^{3}=R^{4}=P h$;

9b: $\mathrm{R}^{2}=\mathrm{Me}, \mathrm{R}^{3}=\mathrm{Ph}, \mathrm{R}^{4}=4-\mathrm{MeOC}_{6} \mathrm{H}_{4}$;

9c: $\mathrm{R}^{2}=\mathrm{Me}, \mathrm{R}^{3}=4-\mathrm{MeC}_{6} \mathrm{H}_{4}, \mathrm{R}^{4}=4-\mathrm{MeC}_{6} \mathrm{H}_{4}$;

9d: $\mathrm{R}^{2}=\mathrm{Me}, \mathrm{R}^{3}=\mathrm{Ph}, \mathrm{R}^{4}=4-\mathrm{BrC}_{6} \mathrm{H}_{4}$;

$\mathrm{TsOH}(20 \mathrm{~mol} \%)$
$10 \mathrm{~h}$ at reflux
in 1,4-dioxane

9e: $\mathrm{R}^{2}=\mathrm{Et}, \mathrm{R}^{3}=4-\mathrm{NO}_{2} \mathrm{C}_{6} \mathrm{H}_{4}, \mathrm{R}^{4}=4-\mathrm{MeC}_{6} \mathrm{H}_{4}$;

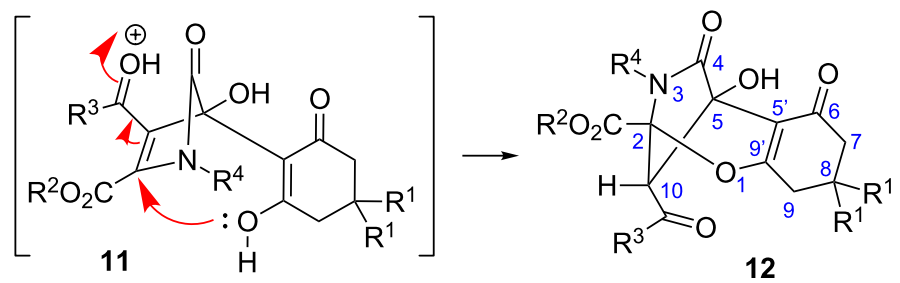

12aa: $R^{1}=R^{2}=M e, R^{3}=R^{4}=P h, 39 \%$;

12ab: $R^{1}=R^{2}=M e, R^{3}=P h, R^{4}=4-M e O C_{6} H_{4}, 32 \%$;

12ac: $R^{1}=R^{2}=M e, R^{3}=R^{4}=4-M C_{6} H_{4}, 36 \%$;

12ad: $R^{1}=R^{2}=M e, R^{3}=P h, R^{4}=4-B_{C} C_{6} H_{4}, 43 \%$;

12ae: $R^{1}=M e, R^{2}=E t, R^{3}=4-N_{2} \mathrm{C}_{6} \mathrm{H}_{4}, \mathrm{R}^{4}=4-\mathrm{MeC}_{6} \mathrm{H}_{4}, 48 \%$;

12bc: $R^{1}=H, R^{2}=M e, R^{3}=R^{4}=4-M e C_{6} H_{4}, 29 \%$;

Scheme 3: Acid-catalyzed spirocyclization of enoles (vinylogous carboxylates) with 5-alkoxycarbonyl-1H-pyrrolediones

Although the reasons for such divergence in the reactivity are not completely understood, one could argue that the lower nucleophilicity of enolates derived from $\mathbf{8}$ as compared to that of enamine 1a could be responsible for this effect. Indeed in this case, the product of 1,2-addition of the $C$-nucleophile to the most reactive keto function and subsequent nucleophilic attack by the $O$-enolate on the conjugate $\mathrm{C}=\mathrm{C}$ bond activated by two electron acceptors could become more preferable as compared to the alternative "normal" pathway, leading to adducts $\mathbf{1 0}$ and involving Michael addition followed by intramolecular transesterification. Remarkably, the resulting bridged products $\mathbf{1 2}$ have reversed regiochemistry as compared to the earlier-described cycloadducts 5 (Scheme 1). It should also be pointed out that all of these products were formed as single 10-endo diastereomers (see Scheme 3 for atom numbering). This configuration was unambiguously confirmed by a single crystal X-ray crystallography of compound 12ab (CCDC 1546062) shown in Figure 1.

Puzzled by this unexpected reactivity, we reasoned that the enolate moiety can be activated towards the desired spirocyclization via conversion of 1,3-diones 8 into mono-hydrazones. Indeed, while mono-imines of these ketones strongly prefer keto-enamine tautomeric form $\mathbf{1 3}$ over enol-imine form $\mathbf{1 4}$ (Scheme 4) [44,45], the corresponding hydrazones have been

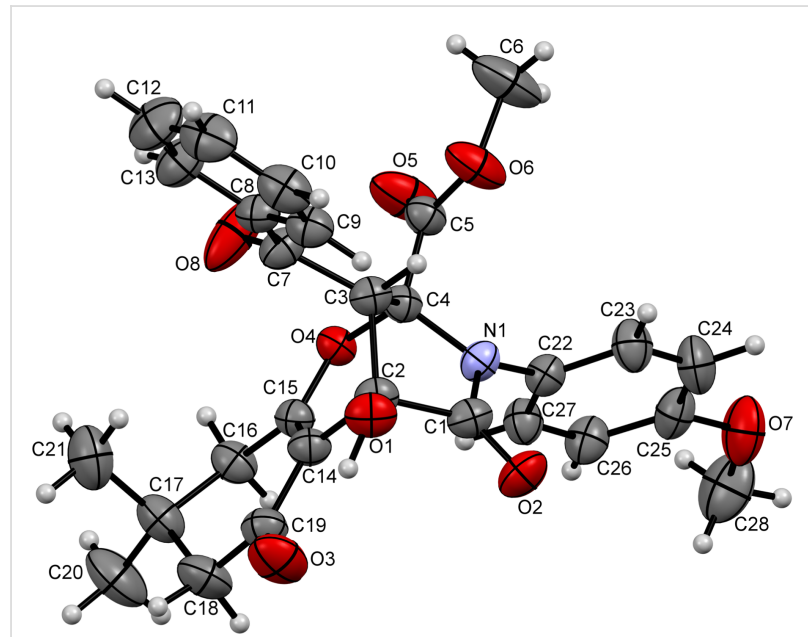

Figure 1: ORTEP drawing of compound 12ab (CCDC 1546062) showing $50 \%$ probability amplitude displacement ellipsoids.

reported to favor enol-hydrazone tautomer 16 (Scheme 4) [4648].

Keeping this in mind we decided to test the reactivity of ketazinones $\mathbf{1 7}$ that were obtained via condensation of cyclohexanediones $\mathbf{8}$ with hydrazone of acetophenone. We anticipated the formation of spirolactones $\mathbf{2 0}$ in this process, resulting from 


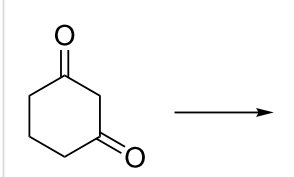

$8 b$

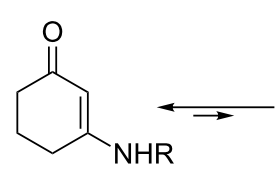

13<smiles></smiles>

14<smiles>[R2]NNC1=CC(=O)CCC1</smiles>

15<smiles>[R20]N=C1C=C(O)CCC1</smiles>

16

Scheme 4: Formation of mono-imines and mono-hydrazones of 1,3cyclohexanediones and tautomeric equilibrium between enol-imine and keto-enamine forms.

intramolecular transesterification involving the enol moiety in tautomeric form 18 (Scheme 5). Surprisingly, an alternative direction of spirocyclization involving the reaction of tautomeric form 19 and affording lactam rings proceeded exclusively. The corresponding spiro[indole-3,2'-pyrroles] 21 were obtained exclusively in good yields (Scheme 5).

The formation of the indoline ring was unambiguously confirmed by the crystal structure of compound 21ab (CCDC 1546063, Figure 2). It seems that the nucleophilicity of the hydrazine moiety prevailed, and the formation of the thermodynamically more favorable amide bond governed the overall direction of this transformation. It should be also taken into

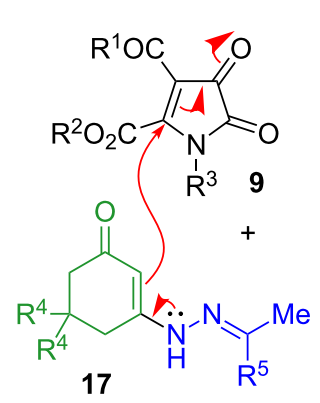

9a: $R^{1}=R^{3}=P h, R^{2}=M e ;$

9b: $\mathrm{R}^{1}=\mathrm{Ph}, \mathrm{R}^{2}=\mathrm{H} ; \mathrm{R}^{3}=4-\mathrm{MeOC}_{6} \mathrm{H}_{4}$;

9c: $\mathrm{R}^{1}=4-\mathrm{MeC}_{6} \mathrm{H}_{4}, \mathrm{R}^{2}=\mathrm{Me} ; \mathrm{R}^{3}=4-\mathrm{MeC}_{6} \mathrm{H}_{4}$;

9d: $\mathrm{R}^{1}=\mathrm{Ph}, \mathrm{R}^{2}=\mathrm{Me} ; \mathrm{R}^{3}=4-\mathrm{BrC}_{6} \mathrm{H}_{4}$;

9e: $\mathrm{R}^{1}=4-\mathrm{NO}_{2} \mathrm{C}_{6} \mathrm{H}_{4}, \mathrm{R}^{2}=\mathrm{Et} ; \mathrm{R}^{3}=4-\mathrm{MeC}_{6} \mathrm{H}_{4}$;

9f: $\mathrm{R}^{1}=4-\mathrm{MeC}_{6} \mathrm{H}_{4}, \mathrm{R}^{2}=\mathrm{Me} ; \mathrm{R}^{3}=\mathrm{Ph}$;

9g: $\mathrm{R}^{1}=\mathrm{Ph}, \mathrm{R}^{2}=\mathrm{Me} ; \mathrm{R}^{3}=4-\mathrm{ClC}_{6} \mathrm{H}_{4}$

17a: $R^{4}=M e, R^{5}=P h$;

17b: $\mathrm{R}^{4}=\mathrm{Me}, \mathrm{R}^{5}=4-\mathrm{MeOC}_{6} \mathrm{H}_{4}$;

17c: $\mathrm{R}^{4}=\mathrm{H}, \mathrm{R}^{5}=4-\mathrm{MeOC}_{6} \mathrm{H}_{4}$.

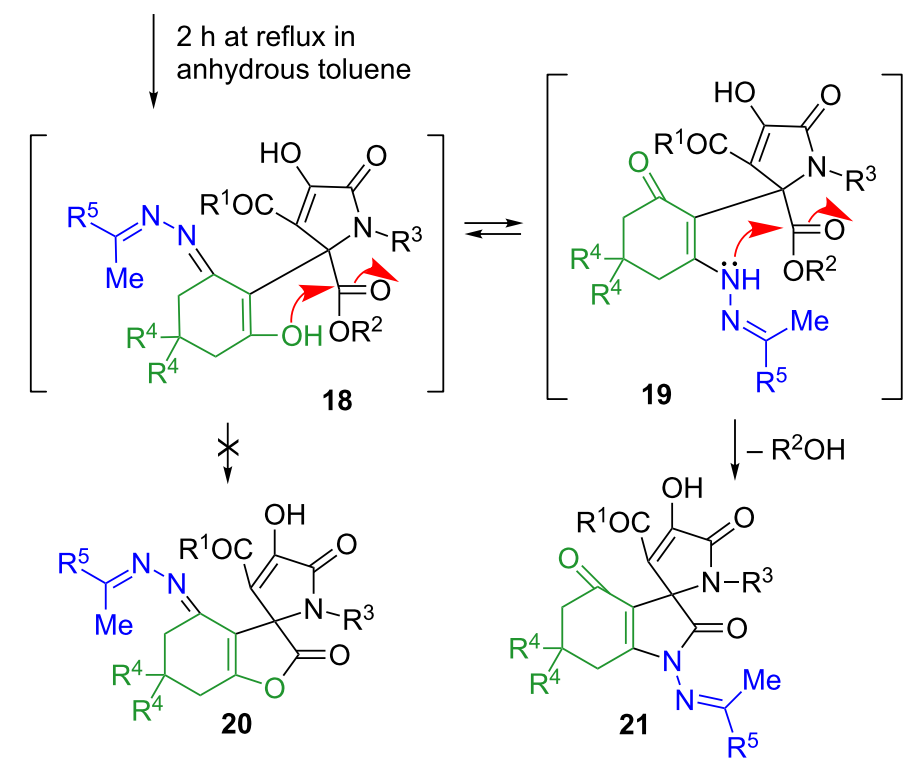

21aa: $R^{1}=R^{3}=R^{5}=P h, R^{4}=M e, 79 \%$;

21ab: $R^{1}=R^{3}=P h, R^{4}=M e, R^{5}=4-M_{e O C} H_{4}, 82 \%$;

21bc: $\mathrm{R}^{1}=\mathrm{Ph}, \mathrm{R}^{3}=4-\mathrm{MeOC}_{6} \mathrm{H}_{4}, \mathrm{R}^{4}=\mathrm{H}, \mathrm{R}^{5}=4-\mathrm{MeOC}_{6} \mathrm{H}_{4}, 70 \%$;

21ca: $R^{1}=R^{3}=4-M_{e} C_{6} H_{4}, R^{4}=M e, R^{5}=P h, 62 \%$;

21da: $R^{1}=P h, R^{3}=4-B r C_{6} H_{4}, R^{4}=M e, R^{5}=P h, 59 \%$;

21eb: $\mathrm{R}^{1}=4-\mathrm{NO}_{2} \mathrm{C}_{6} \mathrm{H}_{4}, \mathrm{R}^{3}=4-\mathrm{MeC}_{6} \mathrm{H}_{4}, \mathrm{R}^{4}=\mathrm{Me}, \mathrm{R}^{5}=4-\mathrm{MeOC}_{6} \mathrm{H}_{4}, 76 \%$;

21fb: $R^{1}=4-M_{e} C_{6} H_{4}, R^{3}=P h, R^{4}=M e, R^{5}=4-M_{e O C} H_{4}, 83 \%$;

21gb: $R^{1}=P h, R^{3}=4-C l C_{6} H_{4}, R^{4}=M e, R^{5}=4-M_{e O C} H_{4}, 76 \%$. 


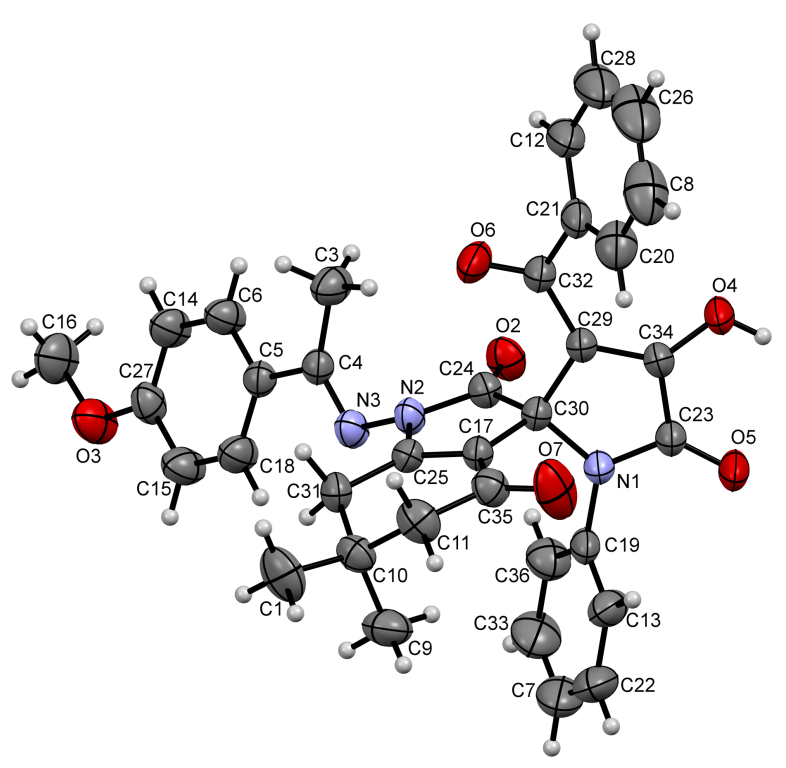

Figure 2: ORTEP drawing of compound 21ab (CCDC 1546063) showing $50 \%$ probability amplitude displacement ellipsoids.

account that, unlike the aforementioned hydrazone structures 15 and 16, ketazinones appear to be more stable in keto-enamine form 17.

We reasoned that the nucleophilicity of the ketazinone moiety can be substantially reduced via incorporation of excessive steric bulk, which ultimately could help us to redirect the course of the reaction towards formation of spirolactones of type $\mathbf{2 0}$. To evaluate this idea, we prepared ketazinones 22 (crystal structure of ketazinone 22a was confirmed by X-ray crystallography (CCDC 1546065, Figure 3)), derived from benzophenone and tested their reactivity with pyrrolediones 9 (Scheme 6). Gratifyingly, this reasoning was correct, as we obtained the corresponding lactones $\mathbf{2 3}$ as the sole products in reasonable yields (Scheme 6).

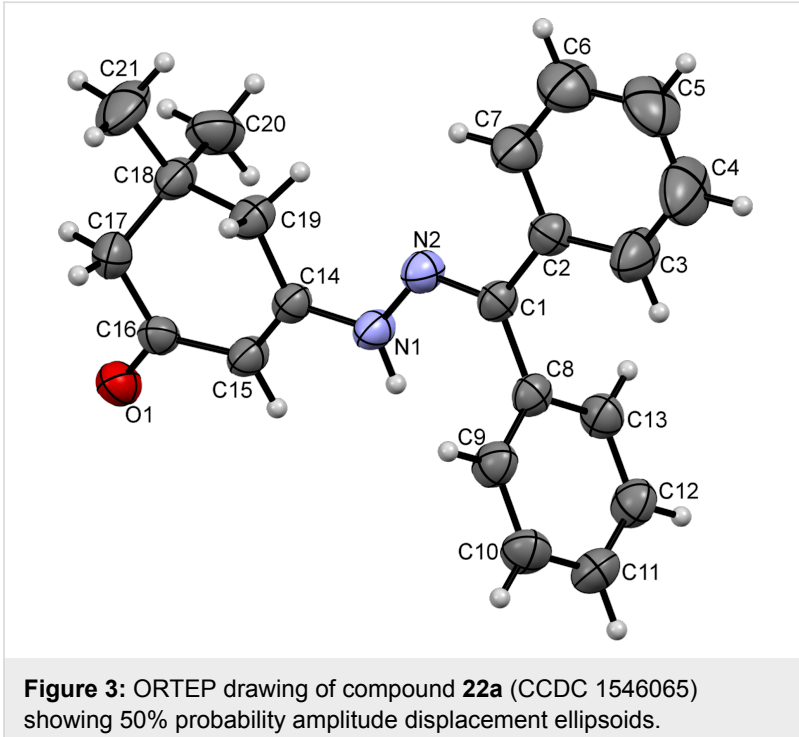<smiles>[R20]C(=O)C1=C(C([R20])=O)N([R3])C(=O)C1=O</smiles>

9a: $R^{1}=R^{3}=P h, R^{2}=M e ;$

9b: $\mathrm{R}^{1}=\mathrm{Ph}, \mathrm{R}^{2}=\mathrm{H} ; \mathrm{R}^{3}=4-\mathrm{MeOC}_{6} \mathrm{H}_{4}$;

9d: $\mathrm{R}^{1}=\mathrm{Ph}, \mathrm{R}^{2}=\mathrm{Me} ; \mathrm{R}^{3}=4-\mathrm{BrC}_{6} \mathrm{H}_{4}$;

9e: $\mathrm{R}^{1}=4-\mathrm{NO}_{2} \mathrm{C}_{6} \mathrm{H}_{4}, \mathrm{R}^{2}=\mathrm{Et} ; \mathrm{R}^{3}=4-\mathrm{MeC}_{6} \mathrm{H}_{4}$;

9g: $\mathrm{R}^{1}=\mathrm{Ph}, \mathrm{R}^{2}=\mathrm{Me} ; \mathrm{R}^{3}=4-\mathrm{ClC}_{6} \mathrm{H}_{4}$;

9h: $\mathrm{R}^{1}=4-\mathrm{BrC}_{6} \mathrm{H}_{4}, \mathrm{R}^{2}=\mathrm{Me} ; \mathrm{R}^{3}=4-\mathrm{MeC}_{6} \mathrm{H}_{4}$.

22a: $R^{4}=M e$;

22b: $R^{4}=H$. 
The crystal structure of compound 23aa (CCDC 1546064) depicted in Figure 4 confirmed the formation of this elusive scaffold.

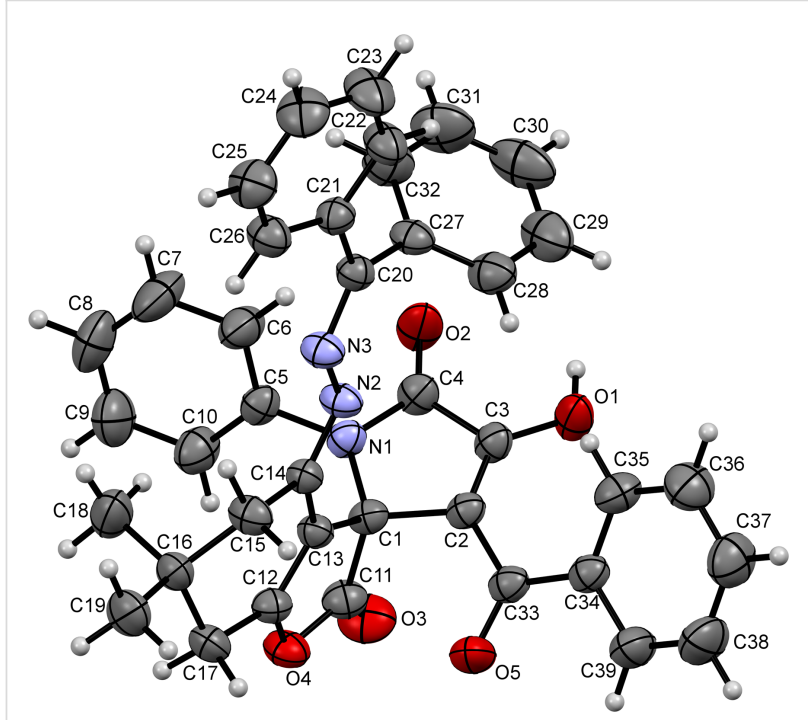

Figure 4: ORTEP drawing of compound 23aa (CCDC 1546064) showing $50 \%$ probability amplitude displacement ellipsoids.

\section{Conclusion}

In conclusion, we discovered a new mode of cyclocondensations with "inverted" regiochemistry of addition, which involved the acid-catalyzed reaction of 5-alkoxycarbonyl-4aroyl-1H-pyrrole-2,3-diones with cyclohexane-1,3-diones and lead to the formation of bridged 2,5-methanobenzo[f][1,3]oxazepines. We also found efficient regiodivergent spirocondensation of the same pyrrolediones with cyclic ketazinones affording the formation of spirocyclic scaffolds with either hydroindoles or hydrobenzofuran moieties. Remarkably, the direction of this condensation can be efficiently switched towards the formation of either of the products by tweaking steric parameters of the employed ketazinones.

\section{Supporting Information}

\section{Supporting Information File 1}

${ }^{1} \mathrm{H}$ and ${ }^{13} \mathrm{C}$ NMR spectral charts and experimental procedures.

[http://www.beilstein-journals.org/bjoc/content/ supplementary/1860-5397-13-218-S1.pdf]

\section{Supporting Information File 2}

$\mathrm{X}$-ray CIF files.

[http://www.beilstein-journals.org/bjoc/content/ supplementary/1860-5397-13-218-S2.zip]

\section{Acknowledgements}

This study was supported by the Russian Foundation for Basic Research (project \#16-43-590613) and by the Ministry of Education and Science of the Russian Federation (project \#4.6774.2017). Financial support in the frame of the Grant from the President of the Russian Federation (project \#MK1657.2017.3) is also gratefully acknowledged (MVD).

\section{ORCID ${ }^{\circledR} \mathrm{iDs}$}

Michael Rubin - https://orcid.org/0000-0002-1668-9311

\section{References}

1. Raghavan, S.; Ravi, A. Org. Biomol. Chem. 2016, 14, 10222-10229. doi:10.1039/C6OB01966H

2. Zhong, S.; Sauter, P. F.; Nieger, M.; Bräse, S. Chem. - Eur. J. 2015, 21, 11219-11225. doi:10.1002/chem.201501199

3. Zhang, W.; Ma, L.; Li, S.; Liu, Z.; Chen, Y.; Zhang, H.; Zhang, G.; Zhang, Q.; Tian, X.; Yuan, C.; Zhang, S.; Zhang, W.; Zhang, C. J. Nat. Prod. 2014, 77, 1887-1892. doi:10.1021/np500362p

4. Diethelm, S.; Schindler, C. S.; Carreira, E. M. Chem. - Eur. J. 2014, 20, 6071-6080. doi:10.1002/chem.201400046

5. Hostetler, G.; Dunn, D.; McKenna, B. A.; Kopec, K.; Chatterjee, S. Chem. Biol. Drug Des. 2014, 83, 149-153. doi:10.1111/cbdd.12240

6. Zhang, F.-M.; Tu, Y.-Q.; Liu, J.-D.; Fan, X.-H.; Shi, L.; Hu, X.-D.; Wang, S.-H.; Zhang, Y.-Q. Tetrahedron 2006, 62, 9446-9455. doi:10.1016/j.tet.2006.07.027

7. Wipf, P.; Rector, S. R.; Takahashi, H. J. Am. Chem. Soc. 2002, 124, 14848-14849. doi:10.1021/ja028603t

8. Padwa, A.; Brodney, M. A.; Dimitroff, M.; Liu, B.; Wu, T. J. Org. Chem. 2001, 66, 3119-3128. doi:10.1021/jo010020z

9. Rigby, J. H.; Cavezza, A.; Heeg, M. J. J. Am. Chem. Soc. 1998, 120, 3664-3670. doi:10.1021/ja974317j

10. Martin, S. F.; Davidsen, S. K. J. Am. Chem. Soc. 1984, 106, 6431-6433. doi:10.1021/ja00333a061

11. Antúnez, D.-J. B.; Greenhalgh, M. D.; Fallan, C.; Slawin, A. M. Z.; Smith, A. D. Org. Biomol. Chem. 2016, 14, 7268-7274. doi:10.1039/C6OB01326K

12. He, Z.-T.; Tian, B.; Fukui, Y.; Tong, X.; Tian, P.; Lin, G.-Q. Angew. Chem., Int. Ed. 2013, 52, 5314-5318. doi:10.1002/anie.201300137

13. Takeda, N.; Ueda, M.; Kagehira, S.; Komei, H.; Tohnai, N.; Miyata, M.; Naito, T.; Miyata, O. Org. Lett. 2013, 15, 4382-4385. doi:10.1021/ol401897u

14. Saliu, F.; Tolppa, E.-L.; Zoia, L.; Orlandi, M. Tetrahedron Lett. 2011, 52, 3856-3860. doi:10.1016/j.tetlet.2011.05.072

15. Bertolini, F.; Pineschi, M. Org. Prep. Proced. Int. 2009, 41, 385-418. doi:10.1080/00304940903240836

16. Barradas, S.; Carreño, M. C.; González-Lopez, M.; Latorre, A.; Urbano, A. Org. Lett. 2007, 9, 5019-5022. doi:10.1021/ol702236e

17. Liu, Q.; Rovis, T. J. Am. Chem. Soc. 2006, 128, 2552-2553. doi:10.1021/ja058337u

18. Crimmins, M. T.; Brown, B. H.; Plake, H. R. J. Am. Chem. Soc. 2006 , 128, 1371-1378. doi:10.1021/ja056334b

19. Ogino, T.; Kurihara, C.; Baba, Y.; Kanematsu, K. J. Chem. Soc., Chem. Commun. 1994, 1979-1980. doi:10.1039/c39940001979

20. Zambias, R. A.; Caldwell, C. G.; Kopka, I. E.; Hammond, M. L. J. Org. Chem. 1988, 53, 4135-4137. doi:10.1021/jo00252a056 
21. Li, J.-L.; Li, Q.; Yang, K.-C.; Li, Y.; Zhou, L.; Han, B.; Peng, C.; Gou, X.-J. RSC Adv. 2016, 6, 38875-38879. doi:10.1039/C6RA06441H

22. Zhang, M.-L.; Yue, D.-F.; Wang, Z.-H.; Luo, Y.; Xu, X.-Y.; Zhang, X.-M.; Yuan, W.-C. Beilstein J. Org. Chem. 2016, 12, 295-300. doi:10.3762/bjoc.12.31

23. Bhanushali, M.; Zhao, C.-G. Tetrahedron Lett. 2012, 53, 359-362. doi:10.1016/j.tetlet.2011.11.056

24. Abd El-Nabi, H. A. Tetrahedron 2002, 58, 135-141. doi:10.1016/S0040-4020(01)01029-8

25. Fabian, W. M. F. J. Org. Chem. 2002, 67, 7475-7482. doi:10.1021/jo026142o

26. Wang, C.; Jia, H.; Zhang, C.; Gao, Z.; Zhou, L.; Yuan, C.; Xiao, Y.; Guo, H. J. Org. Chem. 2017, 82, 633-641. doi:10.1021/acs.joc.6b02659

27. Lu, Y.; Zhou, Y.; Lin, L.; Zheng, H.; Fu, K.; Liua, X.; Feng, X. Chem. Commun. 2016, 52, 8255-8258. doi:10.1039/C6CC03346F

28. Wei, H.-X.; Zhou, C.; Ham, S.; White, J. M.; Birney, D. M. Org. Lett. 2004, 6, 4289-4292. doi:10.1021/ol048197d

29. George, L.; Bernhardt, P. V.; Netsch, K.-P.; Wentrup, C. Org. Biomol. Chem. 2004, 2, 3518-3523. doi:10.1039/b412530d

30. Cobas, A.; Guitian, E.; Castedo, L. J. Org. Chem. 1993, 58, 3113-3117. doi:10.1021/jo00063a034

31. Kappe, C. O.; Kollenz, G.; Wentrup, C. J. Chem. Soc., Chem. Commun. 1992, 485-486. doi:10.1039/c39920000485

32. Ott, W.; Kollenz, G.; Ziegler, E. Synthesis 1976, 546-547. doi:10.1055/s-1976-24119

33. Kollenz, G.; Penn, G.; Ott, W.; Peters, K.; Peters, E.-M.; von Schnering, H. G. Chem. Ber. 1984, 117, 1310-1329. doi:10.1002/cber.19841170406

34. Dagoneau, D.; Xu, Z.; Wang, Q.; Zhu, J. Angew. Chem., Int. Ed. 2016, 55, 760-763. doi:10.1002/anie.201508906

35. Yoshida, Y.; Mohri, K.; Isobe, K.; Itoh, T.; Yamamoto, K. J. Org. Chem. 2009, 74, 6010-6015. doi:10.1021/jo9008645

36. Nimgirawath, S.; Udomputtimekakul, P. Molecules 2009, 14, 917-924. doi:10.3390/molecules 14030917

37. Hosoi, S.; Nagao, M.; Tsuda, Y.; Isobe, K.; Sano, T.; Ohta, T. J. Chem. Soc., Perkin Trans. 1 2000, 1505-1511. doi:10.1039/b001906m

38. Tsuda, Y.; Ohshima, T.; Hosoi, S.; Kaneuchi, S.; Kiuchi, F.; Jun, T.; Sano, T. Chem. Pharm. Bull. 1996, 44, 500-508. doi:10.1248/cpb.44.500

39. Bannikova, Yu. N.; Maslivets, A. N. Chem. Heterocycl. Compd. 2004, 40, 118-119. doi:10.1023/B:COHC.0000023780.19123.5b

40. Silaichev, P. S.; Chudinova, M. A.; Slepukhin, P. A.; Maslivets, A. N. Russ. J. Org. Chem. 2011, 47, 1718-1722. doi:10.1134/S107042801111011X

41. Silaichev, P. S.; Filimonov, V. O.; Slepukhin, P. A.; Maslivets, A. N. Russ. J. Org. Chem. 2012, 48, 561-565. doi:10.1134/S1070428012040173

42. Denislamova, E. S.; Maslivets, A. N. Russ. J. Org. Chem. 2010, 46, 389-393. doi:10.1134/S1070428010030152

43. Dubovtsev, A. Yu.; Silaichev, P. S.; Nazarov, M. A.; Dmitriev, M. V.; Maslivets, A. N.; Rubin, M. RSC Adv. 2016, 6, 84730-84737. doi:10.1039/C6RA16889B

44. Dudek, G. O.; Holm, R. H. J. Am. Chem. Soc. 1962, 84, 2691-2696. doi:10.1021/ja00873a009
45. Zhuo, J.-C. Magn. Reson. Chem. 1997, 35, 21-29. doi:10.1002/(SICI)1097-458X(199701)35:1<21::AID-OMR28>3.0.CO;2I

46. Dubrovay, Z.; Szalay, P. G. J. Mol. Model. 2014, 20, 2293. doi:10.1007/s00894-014-2293-6

47. Guda, R.; Narsimha, S.; Babu, R.; Muthadi, S.; Lingabathula, H.; Palabindela, R.; Yellu, N. R.; Kumar, G.; Kasula, M. Bioorg. Med. Chem. Lett. 2016, 26, 5517-5523. doi:10.1016/j.bmcl.2016.10.006

48. Weigert, F. J. J. Fluorine Chem. 1972, 1, 445-462. doi:10.1016/S0022-1139(00)82966-8

\section{License and Terms}

This is an Open Access article under the terms of the Creative Commons Attribution License

(http://creativecommons.org/licenses/by/4.0), which permits unrestricted use, distribution, and reproduction in any medium, provided the original work is properly cited.

The license is subject to the Beilstein Journal of Organic Chemistry terms and conditions: (http://www.beilstein-journals.org/bjoc)

The definitive version of this article is the electronic one which can be found at: doi: $10.3762 /$ bjoc. 13.218 